\title{
A numerical analysis of pressure drop and particle capture efficiency by rectangular fibers using LB-DE methods
}

\author{
Jianhua Fan • Franck Lominé • Mustapha Hellou
}

\begin{abstract}
In this work, a coupled lattice Boltzmann method (LBM) and discrete element method (DEM) are used to simulate the particle transport and deposition on rectangular fibers of clean filter. The LBM is employed to describe the fluid flow around the fibers, whereas the DEM is used to deal with the particle dynamics. The effects of the Reynolds number, the fiber aspect ratio and the arrangement of fibers (i.e., orientation angle of a fiber) on the pressure drop and capture efficiency are investigated at the initial stage of the filtration process. The quality factor, commonly used to determine the filtration performance, is also studied. The simulation results illustrate that both pressure drop and capture efficiency are dependent on the orientation angle and aspect ratio. The Reynolds number has only a slight influence on the capture efficiency but has a significant effect on the pressure drop for high aspect ratio. A good filter performance can be obtained for square fiber when the orientation angle is $\pi / 4$ from the quality factor standpoint.
\end{abstract}

Keywords lattice Boltzmann method · discrete element method · capture efficiency · rectangular fiber · particle transport

\section{Introduction}

Filtration is an important process for removing particles from a fluid. It is widely applied in many fields, such as water treatment, reactors for catalysis, particle transport in porous media and air purification. Thus, the optimization of the filters is a crucial challenge and should cover fluid and transported particles at the same time. Filtration by fibrous filter has gained great attention among researchers and industries due to its low cost and easy manufacture $[1,2]$. Some mathematical models have been proposed and experimental investigations have been carried out to predict and improve pressure drop and capture efficiency, since they are two important parameters to evaluate the performance of a filter [3]. Fiber filtration involves interaction between transported particles, the fiber and the fluid. Most initial articles on the subject were concerned with circular fibers and used stream function developments to solve fluid velocity field. For example, Kuwabara [4] gave theoretical formula of drag force by solving the velocity field of viscous Stokes flow around a circle. Kuwabara considered a circular outer boundary of the domain. This hypothesis can be valid for solitary fiber or for a weak density of fibers, but fails when the gap between the fibers is smaller than a given threshold. Indeed, in such a case, the outer boundary does not cover all the fluid domain. Lee and Liu [5] based on a boundary layer approach using the Kuwabara flow field combine the diffusion and interception mechanisms together to drive an expression for predicting the single fiber efficiency. Schweers et al. [6] experimentally determined the collection efficiency of single circular fiber and two circular fibers in different arrangements. Augusto et al. [7] used the bounce-back scheme and slip condition to simulate the pressure drop through filters with the OpenLB library. A good agreement is observed by comparing with experimental and numerical results. Some studies include, but are not limited to, pressure drop and capture efficiency of Liu and Wang [8], capture efficiencies due to three mechanisms (Brownian diffusion, interception and inertial impaction) of Stechkina et al. [9], numerical simulation work of Fan et al. [10], Rabiee et al [11], Wang et al. [12] as well as experimental work of Lee and Liu [5].

However, with the development of filter manufacturing technology, researchers recently showed interest for the study of filtration by non-circular fibers $[3,13,14,15]$. In recent years, several researches have been dedicated to square or rectangular fibers. The rectangular fiber is one of the non-circular fibers which have larger specific surface area compared to the circular cross-section fiber with the same volume fraction. Due to this, it is easy to perform higher collection efficiency of particles and to increase mechanical strength, porosity, particle loading capacity for fibers [16]. Fardi and Liu [17] investigated the pressure drop 
with staggered array of rectangular fiber in a viscous fluid flow. Then, they studied the capture efficiency of rectangular fiber dominated by the Brownian diffusion and interception mechanisms [18]. Wang [19], Ouyang and Liu [20] solved the biharmonic equation of 2D Stokes flow across an array of rectangular fibers with different boundary conditions. Wang [19] used a rectangular boundary around each fiber, while Ouyang and Liu [20] used a circular boundary like Kuwabara did. Wang [19] solved the Stokes flow across an array of rectangular fibers and proposed an empirical formula to calculate the drag of a single square fiber. Ouyang and Liu [20] calculated pressure drop of filter with rectangular fiber perpendicular to the flow for various fiber aspect ratios and packing density. Zhu et al. [1] numerically and analytically investigated the impaction-dominated filtration process with rectangular fiber for particulate size much smaller than the one of fiber. The influence of fiber aspect ratio, filter solid fraction, particle size and Stokes number on the collection efficiency of a rectangular fiber has been determined for impaction-dominated filtration. Chen et al. [21] used the control volume method to analyze the effects of fiber aspect ratio, filter volume fraction, size of particles and Reynolds number on the collection efficiency with staggered parallel rectangular fibers. Hosseini and Tafreshi [14] investigated the effect of the fiber's cross-section shape on pressure drop and collection efficiency. They found that the fiber geometry is more important for pressure drop prediction rather than capture efficiency estimation. Most of these studies on rectangular fibers were conducted for a fiber Reynolds number below unity. In this condition, they put in evidence that the drag force applied on rectangular fiber is independent of the fiber Reynolds number.

In order to investigate the filtration process and assess filter performance, it is essential to acquire an accurate fluid velocity field. This can be achieved with numerical simulations. For example, the lattice Boltzmann method (LBM), as an efficient approach to simulate fluid flow, has been widely used for such studies[13, 22, 23, 24, 25, 26, 27]. This method utilizes the Boltzmann equation to describe the fluid phase from a mesoscopic perspective. It is different from conventional numerical methods, which solve the incompressible Navier-Stokes equation [28, 29]. In the LBM, the fluid phase is considered as fictitious fluid particles which can collide and propagate on a lattice. The macroscopic properties (i.e., pressure, fluid density and velocity) can be obtained through the density distribution functions of fluid particles. Compared with conventional methods, LBM demonstrates significant advantages due to its parallel computation and capability to deal with complex boundary conditions [12, 30,31]. As presented in the work of Trunk et al. [32], the fluid and particles detached from the walls of bifurcation are treated using LBM. Jafari et al. [13] computed fluid flow past a square in a channel using the LBM for small particle (diameter less or equal to $10 \mu \mathrm{m}$ ) and Reynolds number larger than 100. In their work, particles transport and deposition are treated with a Lagrangian description, where the presence of particles does not influence the fluid velocity field. Ansari et al. [27] also employed the LBM to calculate the laminar flow field in regular and irregular structure filter to study the transport and deposition of fine particles. They also used a Lagrangian description neglecting the particle influence on fluid due to the small size of particles involved. Concerning the geometry, it can be noted that all these studies are limited to a unique square obstacle in a channel flow.

According to solid particle volume fraction $\phi_{p}[33,34]$, for value of $\phi_{p} \leq 10^{-6}$, the effect of particle on the fluid can be neglected; thus, the one-way coupling could be sufficient. In the second regime $10^{-6}<$ $\phi_{p} \leq 10^{-3}$, the mutual interaction between the fluid and particle need to be considered: two-way coupling. As increased in the volume fraction of particle $\phi_{p}>10^{-3}$, both the fluid-particle interaction and particleparticle interaction should be included, four-way coupling is needed. In many fluid-particle simulations, the fluid and solid phases are generally dealt separately, particle is usually viewed as point without volume [12], the effect of particles on fluid velocity field, and interparticle interactions are often ignored [13, 27, 34]. However, in this work, we consider two-way coupling leading to the action of the fluid on solid particles and reciprocally, though there is a small amount of interparticle interactions. As a first step toward high particle concentrations, a more accurate resolution of the fluid velocity field in the presence of particles is required. This can be achieved through the lattice Boltzmann method (LBM) and the discrete element method (DEM). Interparticle interactions can be simulated with the widely used DEM [35, 36, 37]. Therefore, the coupled methods, LBM for the fluid flow and DEM for interparticle interactions, provide an accurate and predictive tool for simulating fluid-particle problems, as the one treated in this paper [38, 39].

The originality of the present work is to use this coupled LB-DE methods to simulate the transport and deposition of solid particles with geometric boundary in filtration process. Firstly, the hybrid method handles a simple clean filtration problem and demonstrates its ability to reproduce and extend results from previous studies. Then, simulating fluid and particle flow in fibrous filter with rectangular fiber offers a fundamental insights into the physical phenomena in particle-fluid systems. Finally, the pressure drop, capture efficiency and quality factor of periodic arrangement of rectangular fibers with different orientation angles and aspect ratios are simulated at various Reynolds numbers.

In the following section, the numerical method is presented. More specifically, each method is presented separately; then, the coupling between them is presented in turn. In the next section, results of the simu- 
lation of deposition of particles on a rectangular fiber for different aspect ratios and different orientation angles are presented through the analysis of pressure drop, capture efficiency and quality factor.

\section{Numerical method}

2.1 Lattice Boltzmann method for fluid flow

The LB method LBM is based on gas kinetic theory and is applicable to calculate the flow field. The classical D2Q9 model proposed by D'Humières et al. [40] is adopted. In the D2Q9 model, a computational domain is discretized using a square lattice with nine discrete velocities $\mathbf{e}_{i}$ (shown in Fig. 1). The fictitious fluid particles of LBM are constrained to be located at a node and move only along links with nine directions $i$, and collide with each other at the nodes. As presented in Fig. 1, discrete velocities in the D2Q9 model are defined as:

$$
\mathbf{e}_{\mathbf{i}}= \begin{cases}(0,0) & (i=0) \\ C\left(\cos \frac{\pi(i-1)}{2}, \sin \frac{\pi(i-1)}{2}\right) & (i=1, \ldots .4) \\ \sqrt{2} C\left(\cos \frac{\pi(2 i-9)}{4}, \sin \frac{\pi(2 i-9)}{4}\right) & (i=5, \ldots .8)\end{cases}
$$

in which $C$ means the lattice speed given by:

$$
C=h / \mathrm{d} t
$$

$h$ is the lattice spacing and $\mathrm{d} t$ is the time step.

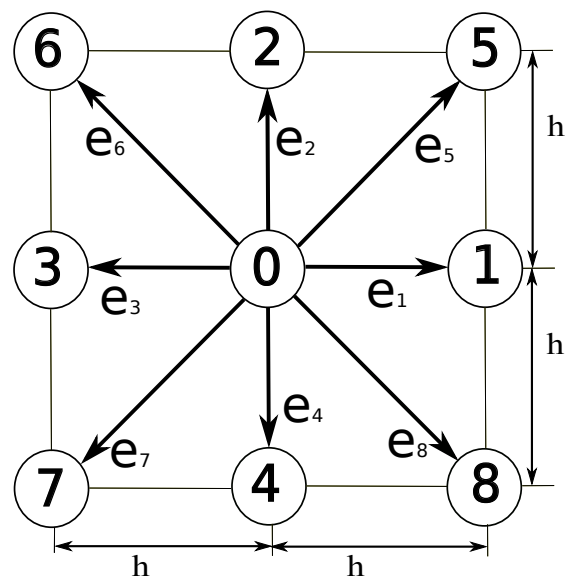

Fig. 1 Discrete velocity in the D2Q9 model

In the LBM, the fundamental notion is particle distribution function $f_{i}$ representing the probability density of fluid particles with velocity $\mathbf{e}_{i}$ along the $i$ th direction at position $x$ and time $t$. The macroscopic properties of the fluid such as mass density $\rho$ and momentum density velocity $\rho \mathbf{u}$ at $(x, t)$ can be determined through moments of $f_{i}$ :

$$
\begin{gathered}
\rho=\sum_{i=0}^{8} f_{i} \\
\mathbf{u}=\frac{1}{\rho} \sum_{i=0}^{8} f_{i} \mathbf{e}_{\mathbf{i}}
\end{gathered}
$$

The principle of LBM consists of two sequential substeps: collision and propagation.

Collision substep:

$$
f_{i}\left(\mathbf{x}, t^{+}\right)=f_{i}(\mathbf{x}, t)+\Delta_{i}
$$

Propagation substep:

$$
f_{i}\left(\mathbf{x}+\mathbf{e}_{\mathbf{i}} \mathrm{d} t, t+\mathrm{d} t\right)=f_{i}\left(\mathbf{x}, t^{+}\right)
$$


The term $f_{i}\left(\mathbf{x}, t^{+}\right)$in Eqs. (5) and (6) is called post-collision term; $t^{+}$is an intermediate time $t<t^{+}<t+\mathrm{d} t$. For any grid node $\mathbf{x}, \mathbf{x}+\mathbf{e}_{\mathbf{i}} \mathrm{d} t$ is its nearest node along the direction $i . \Delta_{i}$ is the collision operator. By introducing a simplified and linearized Bhatnagar-Gross-Krook (called BGK) collision operator [41], the evolution of the density distribution functions is obtained [42]:

$$
f_{i}\left(\mathbf{x}+\mathbf{e}_{\mathbf{i}} \mathrm{d} t, t+\mathrm{d} t\right)=f_{i}(\mathbf{x}, t)-\frac{1}{\tau}\left[f_{i}(\mathbf{x}, t)-f_{i}^{e q}(\mathbf{x}, t)\right]
$$

where $\tau$ is a dimensionless relaxation time. $f_{i}^{e q}$ is the $i$ th equilibrium distribution functions. It is expressed as

$$
f_{i}^{e q}(\mathbf{x}, t)=\kappa_{i} \rho\left(1+\frac{3}{C^{2}} \mathbf{e}_{\mathbf{i}} \cdot \mathbf{u}+\frac{9}{2 C^{4}}\left(\mathbf{e}_{\mathbf{i}} \cdot \mathbf{u}\right)^{2}-\frac{3}{2 C^{2}} \mathbf{u} \cdot \mathbf{u}\right)
$$

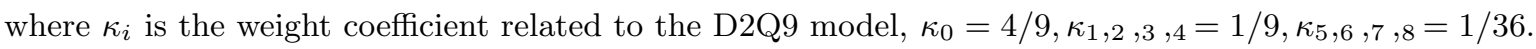

The kinematic viscosity $\nu$ and time step $\mathrm{d} t$ are related to the lattice velocity $C$, dimensionless relaxation time $\tau$ and lattice spacing $h$. The equations for calculating kinematic viscosity and time step are given by:

$$
\begin{aligned}
\nu & =\frac{1}{3}\left(\tau-\frac{1}{2}\right) C h \\
\mathrm{~d} t & =\frac{1}{3 \nu}\left(\tau-\frac{1}{2}\right) h^{2}
\end{aligned}
$$

One of the main assumptions of the LBM approach used in this work consists in considering a small compressibility of the fluid. Hence, its pressure is calculated by the following state law:

$$
p=C_{s}^{2} \rho
$$

where $C_{s}$ is the sound speed, defined as $C_{s}=C / \sqrt{3}$. The value of the Mach number is limited to $M \leq 0.1$, which ensures the compressibility error $M^{2}<1 \%$.

As macroscopic parameters, velocity and pressure are derived from the density distribution functions $f_{i}$, they cannot be imposed directly on the system boundaries. The boundary condition method proposed by Zou and He [43] is applied on the system. At the interface of solid and fluid, bounce-back on the link between nodes $[44,45]$ is considered to ensure the no-slip boundary condition on the solid surface.

\subsection{Discrete element method for particle interactions}

The discrete element method (DEM) introduced by Cundall and Strack [35] is employed to deal with interactions between solid particles in contact. In the DEM, particles are allowed to slightly overlap among each other during their contact. When two particles $a$ and $b$ located at positions $\mathbf{r}_{\mathbf{a}}$ and $\mathbf{r}_{\mathbf{b}}$ contact each other, the overlap is $\chi_{n}=R_{a}+R_{b}-\left(\mathbf{r}_{\mathbf{a}}-\mathbf{r}_{\mathbf{b}}\right) \cdot \mathbf{n}$, where $R_{a}$ and $R_{b}$ are particle radius and $\mathbf{n}$ is a unit vector normal to the contact line in 2D (or contact plane in 3D). However, seeking of interactions between particles is the most CPU time-consuming part of the method. In order to avoid this consumption, a doubly linkedlist method [36] is implemented to optimize interaction search. We used "linear spring dashpot" (LSD) model $[37,46]$ to calculate the normal force $F_{n}$ and an extension method proposed by Cundall and Strack [35] to compute the tangential force $F_{s}$ respectively. The expressions are given by:

$$
\begin{gathered}
F_{n}=K_{n} \chi_{n}+\gamma_{n} \dot{\chi_{n}} \\
F_{s}=F_{s}+\Delta F_{s}
\end{gathered}
$$

where $K_{n}$ is the normal stiffness coefficient of the interaction and $\gamma_{n}$ is a damping coefficient. $\dot{\chi}_{n}$ is the time derivative of $\chi_{n}$. In Eq. (13), $F_{s}$, computed with a shear force increment $\Delta F_{s}$, is initialized to zero when the contact is formed. The $\Delta F_{s}$ can be calculated by accumulating the path of moving contact point between the two particles in contact as follows:

$$
\Delta F_{s}=-K_{s} \Delta \chi_{s}
$$

where $K_{s}$ represents the interaction shear stiffness and $\Delta \chi_{s}$ is the increment of the tangential relative displacement of the particles contact. Tangential sliding occurs if

$$
\left|F_{s}\right|>\min \left(\lambda_{a}, \lambda_{b}\right)\left|F_{n}\right|
$$

where $\lambda_{a}$ and $\lambda_{b}$ are particle friction coefficients. 
For each solid particle, we use the contact law described above to calculate the total contact force $F_{c}$ which is equal to the summation of contact force over all interacting partners. At each time step of the DE method, the new position and velocity of each solid particle are deduced from the explicit time integration of Newton's second law:

$$
m_{p} \frac{\mathrm{d} \mathbf{u}_{p}}{\mathrm{~d} t}=\mathbf{F}_{c}+\mathbf{F}_{h}
$$

where $m_{p}$ and $\mathbf{u}_{p}$ are, respectively, particle mass and velocity. $F_{h}$ is the hydrodynamic force which will be expressed in Sect. 2.3. Torques applied to the solid particle are computed in the same way by integrating the equation

$$
\mathbf{T}_{c}+\mathbf{T}_{h}=J \frac{\mathrm{d} \varpi_{\mathbf{p}}}{\mathrm{d} t}
$$

where $\mathbf{T}_{c}$ is the torque resulting from the inter-particle forces, $\mathbf{T}_{h}$ the hydrodynamic torque (see Sect. 2.3) and $J$ the moment of inertia of particles and $\varpi_{\mathbf{p}}$ the angular velocity. The time integration is performed using Verlet's algorithm [47].

\subsection{LB-DE coupling}

The hydrodynamic force and torque determined from LBM are added to contact forces in the DEM. Then, position and velocity of each solid particle are calculated. Thus, a subcycling scheme is needed in the coupled LB-DE procedure. The DEM time step $\mathrm{d} t_{\mathrm{DE}}$ is generally smaller than the LBM time step $\mathrm{d} t$. As a consequence, a number of DEM loops should be included in a single LBM cycle, which reduced a new value $\mathrm{d} t_{\mathrm{DE}}^{\prime}=\mathrm{d} t / n$, where $n$ is an integer greater or equal to $\mathrm{d} t_{\mathrm{DE}} / \mathrm{d} t . n$ substeps of DEM are performed during one computational step of LBM. Generally, the subcycling scheme works well for small $n$ [38]; however, further investigations for large $n$ are required to understand its behavior. As discussed in Ref. [39], to not alter LB-DE coupling, we choose $n$ values smaller or equal to 3 .

Solid particles are viewed as objects with geometric boundaries, and they are mapped on LBM lattice. During the propagation phase of the LBM, a density distribution function migrates from one lattice node to its nearest node along the direction $i$. The nodes belonging to an obstacle which are the closest ones to its external surface are called "Solid Boundary" (SB) nodes. All the fluid nodes located outside the obstacle, with at least one direction pointing toward a SB node, are called "Fluid Boundary" (FB) nodes. Then, between a SB node and a FB node, we introduce a boundary link. Here we use the "mid-plane bounce-back" boundary condition on the boundary link to describe the transfer of momentum between a $\mathrm{SB}$ and a FB node [44]. At the end of propagation step, the fluid particles reflect along a boundary link $\sigma i$ (pointing from fluid to solid nodes) at mid-distance. Reflected distribution functions are given by [44]:

$$
\begin{aligned}
& f_{-\sigma i}\left(\mathbf{x}_{F B}, t+\mathrm{d} t\right)=f_{\sigma i}\left(\mathbf{x}_{F B}, t^{+}\right)-2 \alpha_{i} \mathbf{u}_{\mathbf{s}} \cdot \mathbf{e}_{\mathbf{i}} \\
& f_{\sigma i}\left(\mathbf{x}_{S B}, t+\mathrm{d} t\right)=f_{-\sigma i}\left(\mathbf{x}_{S B}, t^{+}\right)+2 \alpha_{i} \mathbf{u}_{\mathbf{s}} \cdot \mathbf{e}_{\mathbf{i}}
\end{aligned}
$$

where $-\sigma i$ simply denotes the opposite direction of $\sigma i \cdot \mathbf{x}_{S B}=\mathbf{x}_{F B}+\mathbf{e}_{\sigma \mathbf{i}} \mathrm{d} t, \alpha_{i}=3 \kappa_{i} \rho / C^{2}$. $\mathbf{u}_{\mathbf{s}}$ is the boundary velocity at the middle of the boundary link $\sigma$. It can be expressed as $\mathbf{u}_{\mathbf{s}}=\mathbf{u}_{\mathbf{p}}+\varpi_{\mathbf{p}} \wedge \mathbf{r}_{\mathbf{c}}$, where $\mathbf{u}_{\mathbf{p}}$ and $\varpi_{\mathbf{p}}$ are the translational and angular velocities, respectively, at the center of mass of the solid obstacle. $\mathbf{r}_{\mathbf{c}}=\mathbf{x}_{F B}+\mathbf{e}_{\boldsymbol{\sigma} \mathbf{i}} \mathrm{d} t / 2-\mathbf{x}_{\mathbf{c}}$ is the vector joining the center of mass $\mathbf{x}_{\mathbf{c}}$ of the solid obstacle to the middle of the boundary link.

We can determine the fluid force and torque applied on an obstacle by calculating the momentum exchange over all the boundary links of the obstacle [39]:

$$
\begin{gathered}
\mathbf{F}_{h}\left(t+\frac{1}{2} \mathrm{~d} t\right)=\sum_{\sigma i} 2 \frac{\Omega}{\mathrm{d} t}\left[f_{\sigma i}\left(\mathbf{x}, t^{+}\right)-\alpha_{i} \mathbf{u}_{\mathbf{s}} \cdot \mathbf{e}_{\mathbf{i}}\right] \mathbf{e}_{\sigma i} \\
\mathbf{T}_{h}\left(t+\frac{1}{2} \mathrm{~d} t\right)=\sum_{\sigma i} \mathbf{T}_{\sigma}\left(\mathbf{x}, t+\frac{1}{2} \mathrm{~d} t\right)
\end{gathered}
$$

where $\Omega$ is the cell lattice surface in $2 \mathrm{D}$ (or cell lattice volume for the $3 \mathrm{D}$ case). To be time consistent, hydrodynamic forces and torques applied on obstacles are obtained by an average over two consecutive LBM time steps:

$$
\mathbf{F}_{h}(t)=\frac{1}{2}\left[\mathbf{F}_{h}\left(t+\frac{1}{2} \mathrm{~d} t\right)+\mathbf{F}_{h}\left(t-\frac{1}{2} \mathrm{~d} t\right)\right]
$$




$$
\mathbf{T}_{h}(t)=\frac{1}{2}\left[\mathbf{T}_{h}\left(t+\frac{1}{2} \mathrm{~d} t\right)+\mathbf{T}_{h}\left(t-\frac{1}{2} \mathrm{~d} t\right)\right]
$$

During the DEM loops, the hydrodynamic forces and torques applied on particles remain unchanged between two LBM consecutive time steps. Then, Eqs. (16) and (17) can be solved. New positions and velocities of the solid particles are obtained.

\section{Numerical simulation for filtration process of filter}

A schematic view of a periodic cell of the filter is illustrated in Fig. 2. The fiber, represented by its rectangular cross section, is located in the center of a fluid domain of width $H$ and length $L$. This domain is also the computational one. The fiber can be oriented with angle $\theta$ relatively to $x$-axis. $w$ and $b$ are, respectively, the width and height of the fiber.

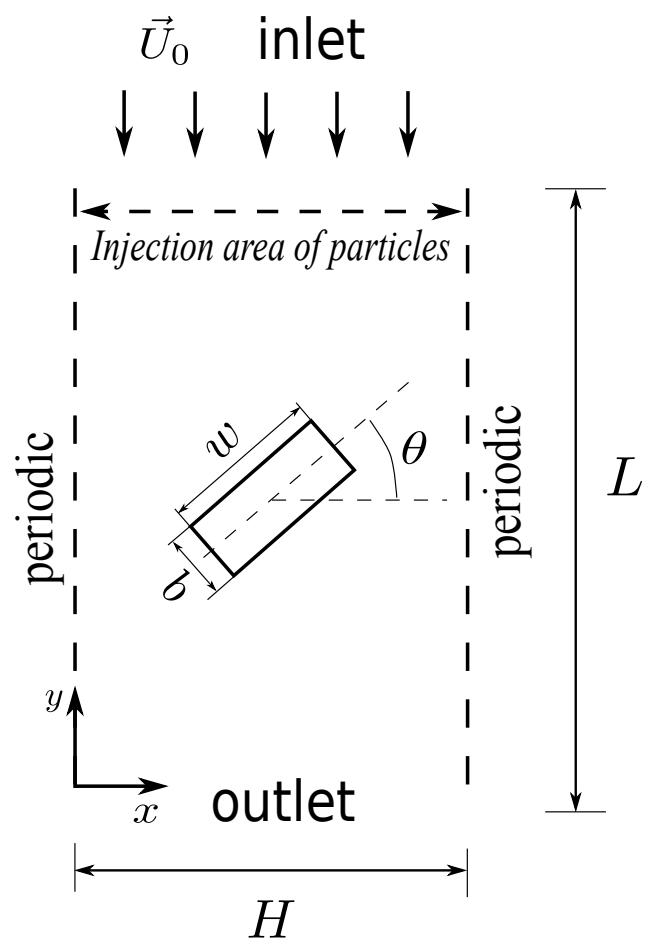

Fig. 2 Schematic view of the computational domain for simulating the flow field around a rectangular fiber

A constant velocity $\mathbf{U}_{0}$ is applied at the inlet of the computational domain. At the outlet, we have imposed $\partial u_{x}(x, 0) / \partial y=0$ and $\partial u_{y}(x, 0) / \partial y=0$, where $u_{x}$ and $u_{y}$ are components of velocity vector $\mathbf{U}_{0}$, respectively, along $x$ and $y$ directions. Both left and right sides of computational domain are periodic boundary conditions for both fluid and particles. After establishment of steady state, particles are randomly injected into the domain on a line at the inlet (see Fig. 2). Since the particle size used here is $d_{p}=30 \mu \mathrm{m}$, the Brownian diffusion is relatively weak, and the Brownian force on a particle is far less than the drag force exerted by the fluid [12]. For this reason, the Brownian diffusion is not considered in this work. The initial velocity of each injected particle is equal to the fluid velocity at this location.

The filtration process usually includes two stages: initial and aging stages [48]. At initial stage, the captured particles inside the filter is relatively small. The influence of deposited particles on the properties of the fiber is negligible, fiber shape does not change with time, and filter is regarded as a clean one. Thus, it is reasonable to assume that particles are removed from the flow once they are captured by the fiber at this stage of filtration $[25,26]$. However, at the aging stage, a large number of particles will deposit on the surface of the fiber, which change the shape of fiber and lead to the progressive clogging of filter and the increase of the pressure drop. In this study, we focused on the initial stage of the filtration process. Moreover, particles that are not captured by the fiber are removed when they escape from the outlet. The capture efficiency can be obtained as follows:

$$
\eta=\left(N_{\text {inject }}-N_{\text {escape }}\right) / N_{\text {inject }}
$$


Table 1 Model parameters used in the simulations

\begin{tabular}{lll}
\hline Channel length, $L$ & 0.0017 & $(\mathrm{~m})$ \\
Channel height, $H$ & 0.0009 & $(\mathrm{~m})$ \\
Fluid velocity, $U_{0}$ & $0.157,0.314,0.471,0.628,0.758$ & $(\mathrm{~m} / \mathrm{s})$ \\
Fluid kinetic viscosity, $\nu$ & $1.57 \times 10^{-5}$ & $\left(\mathrm{~m}^{2} / \mathrm{s}\right)$ \\
Fluid density, $\rho$ & 1.2 & $\left(\mathrm{~kg} / \mathrm{m}^{3}\right)$ \\
Relaxation time, $\tau$ & 1 & $(-)$ \\
Time step, d $t$ & $3.45 \times 10^{-8}$ & $(\mathrm{~s})$
\end{tabular}

where $N_{\text {inject }}$ and $N_{\text {escape }}$ represent, respectively, the number of solid particles injected at the inlet and removed from the outlet.

The grid resolution for LBM, different values of $d_{p} / h=8,10,12,14,16$ are examined. The drag coefficient is compared with the result obtained from commercial software Comsol. It is found that good agreement can be observed for $d_{p} / h \geq 10$ and the number of nodes does not affect the drag coefficient. In this work, we fix grid resolution of LBM here $d_{p} / h \approx 16$, which is good enough to compute a reliable flow field and hydrodynamic force. Other parameters used in the simulations are presented in Table 1.

In the following part, we investigate the effects of Reynolds number $R e$ of the fiber, aspect ratio $w / b$ and orientation angle of the fiber $\theta$ on the pressure drop and capture efficiency of the filter. The Reynolds number is defined as $R e=U_{0} D_{h} / \nu$, where $D_{h}$ is the hydraulic diameter of the fiber equal to $D_{h}=$ $4 S / P=2 w b /(w+b), S$ is cross-section area and $P$ is the perimeter. Four different values of aspect ratio $(w / b=1,2,3,4)$ are used in the simulations while keeping $D_{h}$ constant. Five orientation angles $(\theta=0, \pi / 8, \pi / 4,3 \pi / 8, \pi / 2)$ are studied for each aspect ratio. The solid volume fraction of the fibers is computed as $\beta=b w /(L H)$.

\subsection{Pressure drop for clean fiber}

The pressure drop $\Delta p$ between inlet and outlet of the periodic cell, which is related to drag force $F_{D}$ on a unit length of single fiber, is a key parameter to evaluate filtration performance [8]. The determination of the dimensionless drag force $F^{*}=F_{D} /\left(\mu U_{0}\right)$ permits to calculate the pressure drop, where $\mu$ is the dynamic viscosity, with the following relation:

$$
\frac{\Delta p}{L}=\frac{F_{D} \beta}{b w}
$$

Ouyang and Liu [20] determined the dimensionless drag force for a rectangular fiber with $\theta=0$ in Stokes flow regime. It is worth to note that the volume fraction has also an influence on the dimensionless drag force. In order to compare with these results and to validate our model, we have plotted in Fig. 3 the dependency of the dimensionless drag force, obtained with our program, on the volume fraction of fiber for different aspect ratios $w / b$ and $R e=0.2$ and more generally $R e<1$ [20]. Figure 3 shows a good agreement and demonstrates that our model can be used to simulate the pressure drop of a filter with rectangular fiber for low Reynolds number.

In addition to the volume fraction, the aspect ratio, orientation angle of rectangular fiber as well as Reynolds number influence the pressure drop. In previous works, many researchers generally choose the Reynolds number $<1[11,16]$. As $R e<1$, Liu and Wang [8] found that the dimensionless drag force is independent of Reynolds number for circular fiber. Furthermore, Fardi and Liu [17] concluded also that $F_{D}$ is relatively independent of $R e$ for rectangular fiber. In this study, we focus on the study of the relationship between dimensionless force and Reynolds number for the case of $R e>1$. To vary the Reynolds number, we have chosen to vary the fluid velocity at the inlet.

Figure 4 shows the dimensionless drag force $F^{*}$ as a function of Reynolds number, varying from 2 to 10, with four typical aspect ratios and $\theta=0$. As presented in Fig. $4, F^{*}$ is influenced by Reynolds number and increased with it in the region of $2 \leq R e \leq 10$. When $R e$ increases, the growth rate of $F^{*}$ becomes gradually enhanced for aspect ratio $(w / b)$ from 1 to 4 . Thus, Reynolds number has significant effect on the pressure drop for large value of aspect ratio. We know that a lower pressure drop across a filter is more favorable. Here at a fixed Reynolds number, it is obtained for rectangular fiber with a small aspect ratio (i.e., $w / b=1)$.

The streamlines around rectangular fiber with aspect ratio of $w / b=2$ and different orientation angles are presented in Fig. 5. It is obviously observed that the orientation angle of fiber modifies the distribution of the flow field, which furthermore influences the pressure drop. The streamlines near the fiber change remarkably as the orientation angle decreases. Figure 8 shows the relationship between dimensionless drag force and orientation angle with different aspect ratios. Complementary to this figure, Fig. 6 shows the evolution of the windward area $l_{c}$, defined as: 


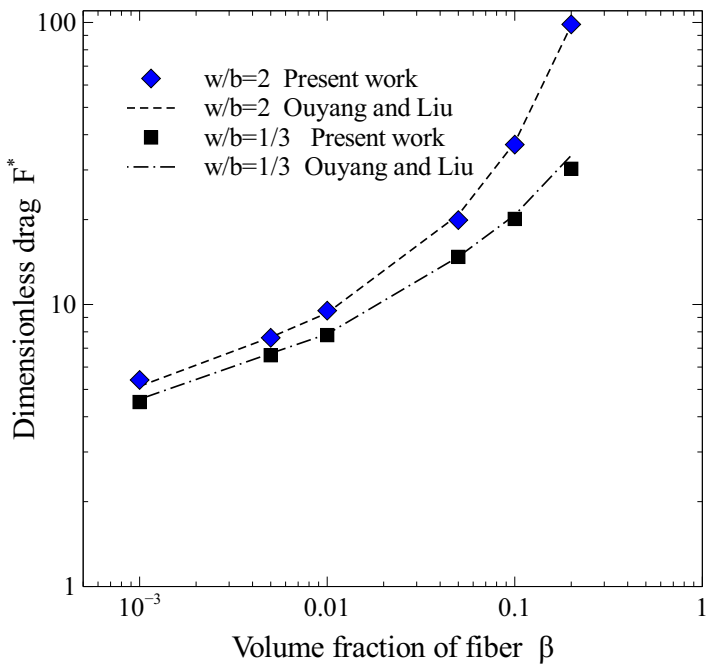

Fig. 3 Dimensionless drag force as a function of volume fraction, LB simulation results are presented and compared with the work of Ouyang and Liu [20].

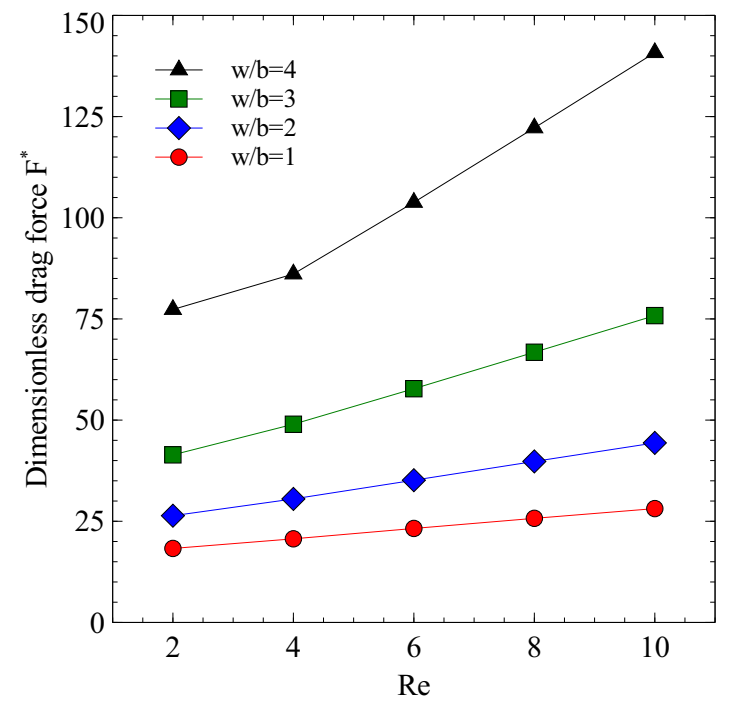

Fig. 4 Dimensionless drag force as a function of fiber Reynolds number for $\theta=0$.

$$
l_{c}=w \cos \theta+b \sin \theta
$$

and represented in Fig. 7. For $l_{c}$, we vary $w$ and $b$ in order to keep $D_{h}$ fixed in the simulation. The maximum value of $l_{c}$ for each aspect ratio is also plotted. Figure 8 shows the dimensionless drag force varying with $\theta$. It illustrates that when the aspect ratio $w / b=1$ (i.e., square), $F^{*}$ is larger for $\theta=\pi / 4$ than for the other angles due to the largest windward area in comparison with the other angle values.

The maximum windward area $l_{c}$ is $\arctan b / w$. However, $F^{*}$ decreases with $\theta$ for another aspect ratios $w / b=2,3,4$, the larger $w / b$ is, the faster the $F^{*}$ decrease is. It can also be noted that $F^{*}$ at $\theta=\pi / 2$ is lower than that at $\theta=0$ for $w / b=2,3,4$. As observed in Fig. 6 , the reason is that the windward area of $\theta=\pi / 2$ is much smaller than that of $\theta=0$. Therefore, as $\theta$ increases, the windward area of the rectangular fiber decreases, which allows the fluid to pass through the filter more easily. It can also be noted, in Fig. 6 that the values of $l_{c}$ are quite similar for $\theta=3 \pi / 8$ and $\pi / 2$, leading to quite similar values of drag force as observed in Fig. 6. Based on this analysis, it is concluded that a larger $\theta$ with a smaller windward area of rectangular fiber is more stable to obtain a lower pressure drop. Figure 9 presents the same simulations results of Fig. 8 in terms of cross section $l_{c}$ for all aspect ratios investigated. It illustrates that drag force increases with $l_{c}$. In this analysis, we present the relationship between $F^{*}$ and $\theta$ (or $l_{c}$ ) in the case where $b \leq w$. However, for the case of $b>w$ (i.e., $w / b=1 / 2,1 / 3,1 / 4$ ), symmetrical results about $\theta=\pi / 4$ with those of Fig. 8 can be obtained, which are not demonstrated in this work. 


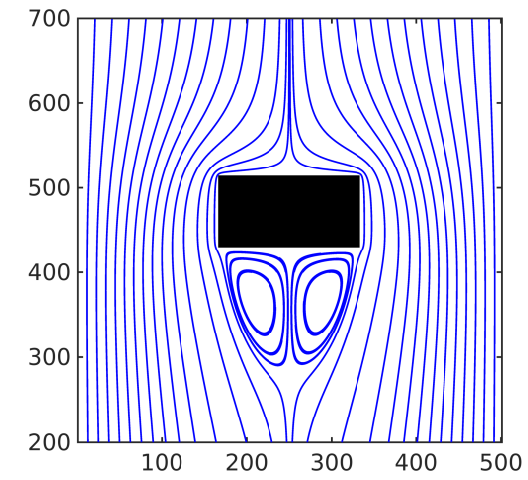

a)

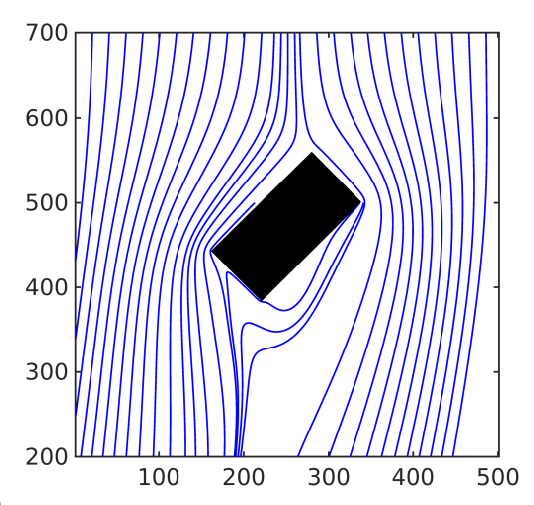

b)
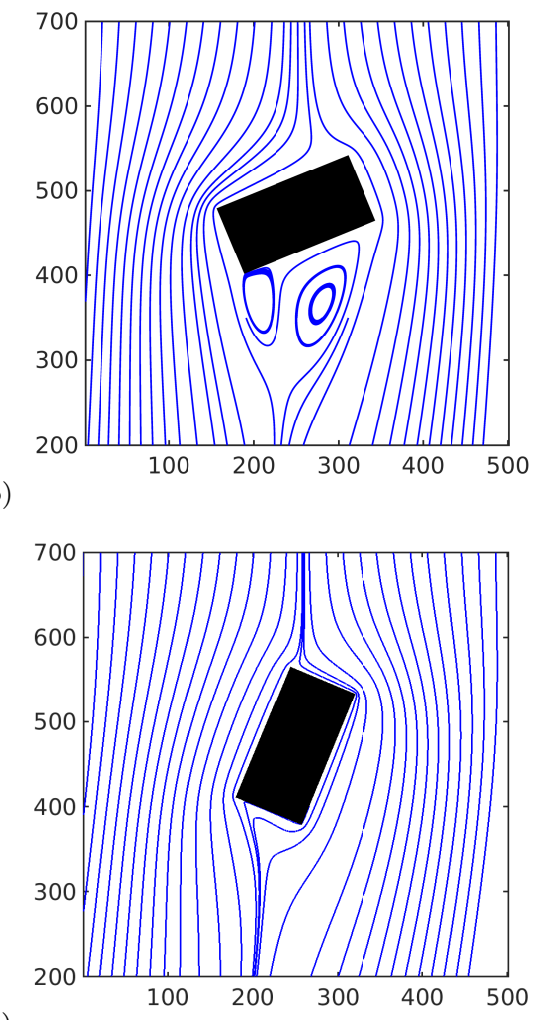

d)

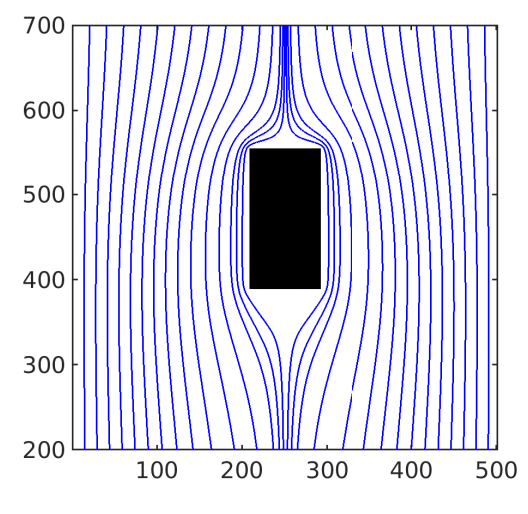

Fig. 5 Streamlines around rectangular fiber with an aspect ratio of $w / b=2$ and orientation angles. a) $\theta=0$, b) $\theta=\pi / 8$, c) $\theta=\pi / 4$, d) $\theta=3 \pi / 8$, e) $\theta=\pi / 2$. Re $=8$ for all configurations.

\subsection{Capture efficiency}

Capture efficiency, defined in Eq. (23), is another important parameter used to assess the filter performance. The capture efficiency obtained using LB-DE methods has been validated by comparison of empirical correlation for single circular fiber in our previous work [49]. Good agreement is observed between the results of LB-DE methods and empirical correlation. Thus, the LB-DE methods developed in this work can be used to simulate the capture efficiency. In the present simulations, the total number of solid particles is 500 and 10 particles are randomly injected every $0.00016 \mathrm{~s}$ at the whole inlet, as illustrated in Fig. 2, once the flow field reaches a steady state. The velocity and trajectory of solid particles depend obviously on the fluid velocity field, whose regime is characterized by Reynolds number. Thus, the relationship between capture efficiency and Reynolds number is sought in Fig. 10. It is shown that the capture efficiency varies slightly with the Reynolds number from 2 to 10 for a fixed aspect ratio and orientation $\theta=0$. The reason is that particles are mainly dominated by the inertial impaction, which means that particles deviate from the flow streamlines at first, and after several time steps, they do not follow them any more. For $R e=10$, particles move straight ahead from inlet to outlet since they have a strong inertial force. For this case, the Reynolds number does not have a significant effect on capture efficiency. In the region of $2 \leq R e \leq 10$, the 


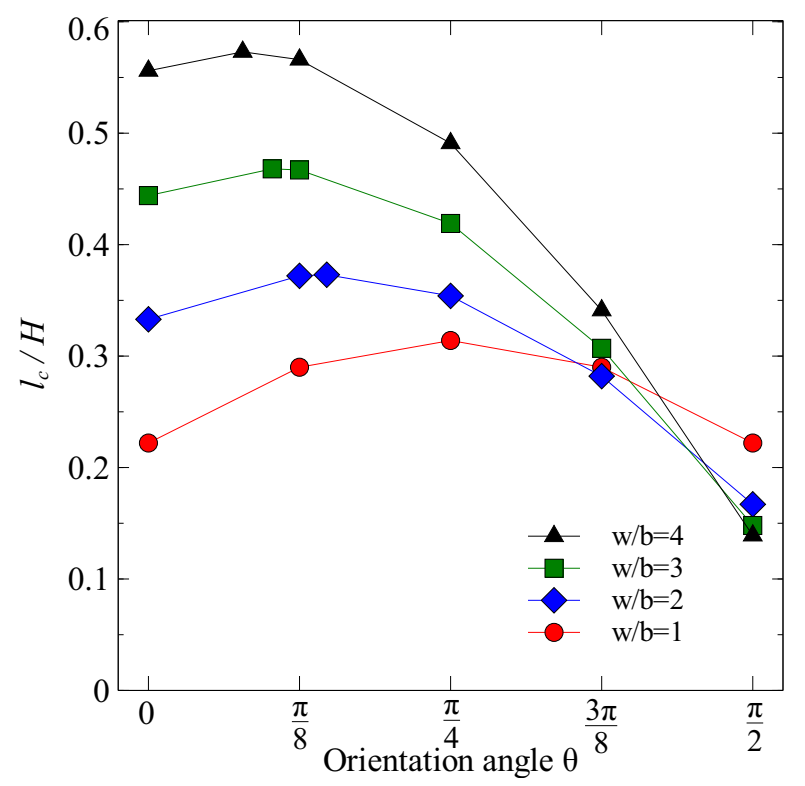

Fig. 6 Windward area with orientation angle $\theta$.

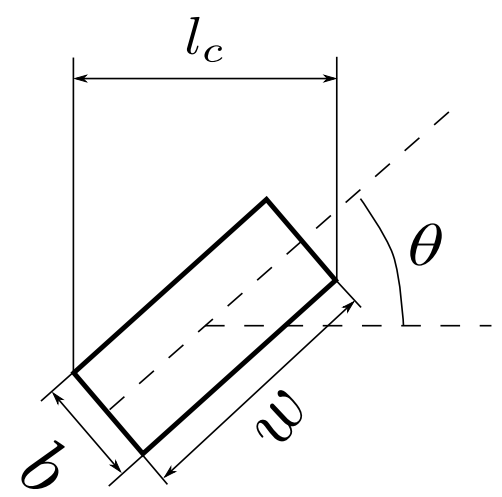

Fig. 7 The cross-section area of rectangular fiber

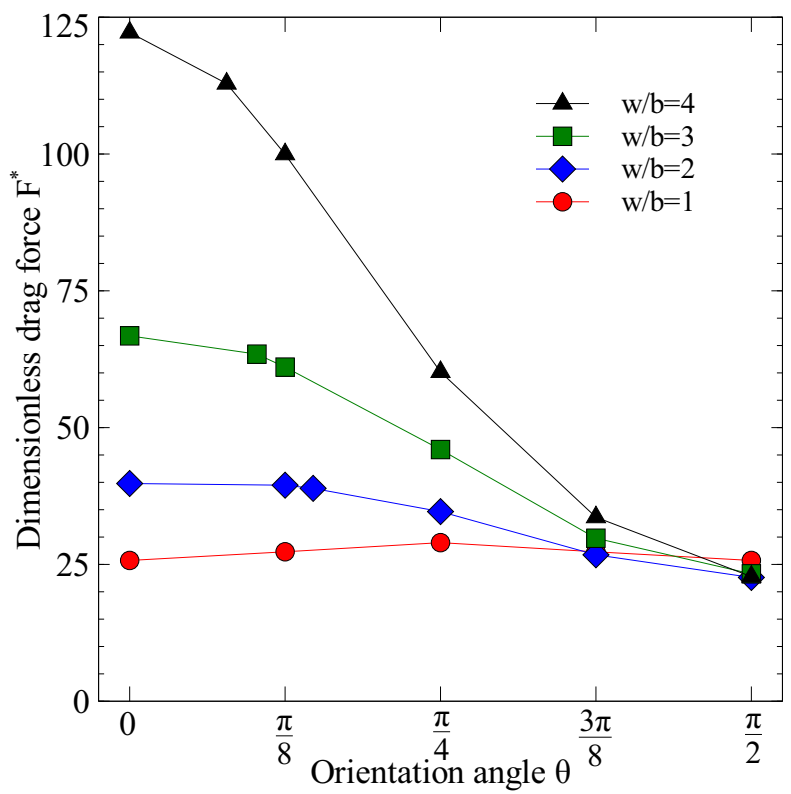

Fig. 8 Dimensionless drag force as a function of orientation angle, $R e=8$. 


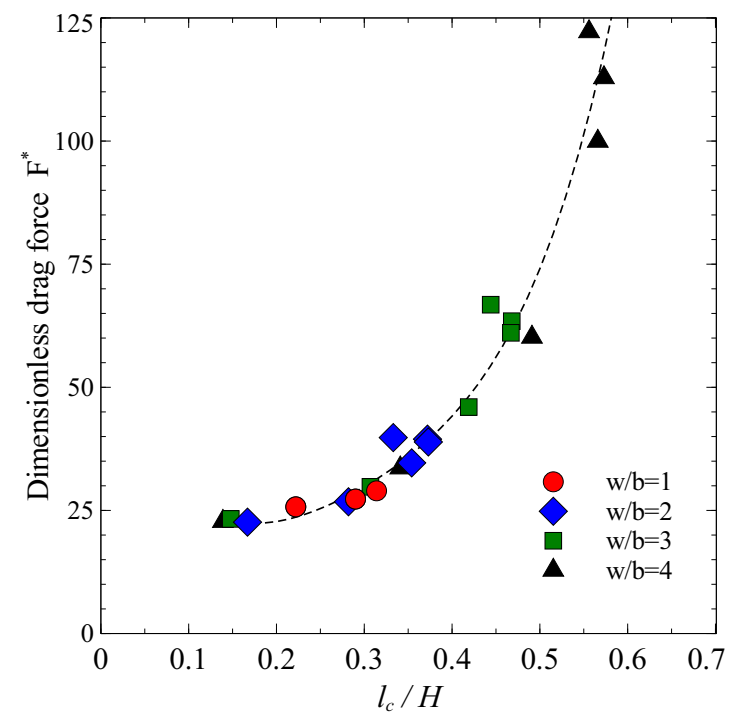

Fig. 9 Dimensionless drag force as a function of cross section $l_{c}, R e=8$.

rectangular fiber with large aspect ratio (i.e., large $w$ ) is more stable to obtain a high capture efficiency due to its large windward area.

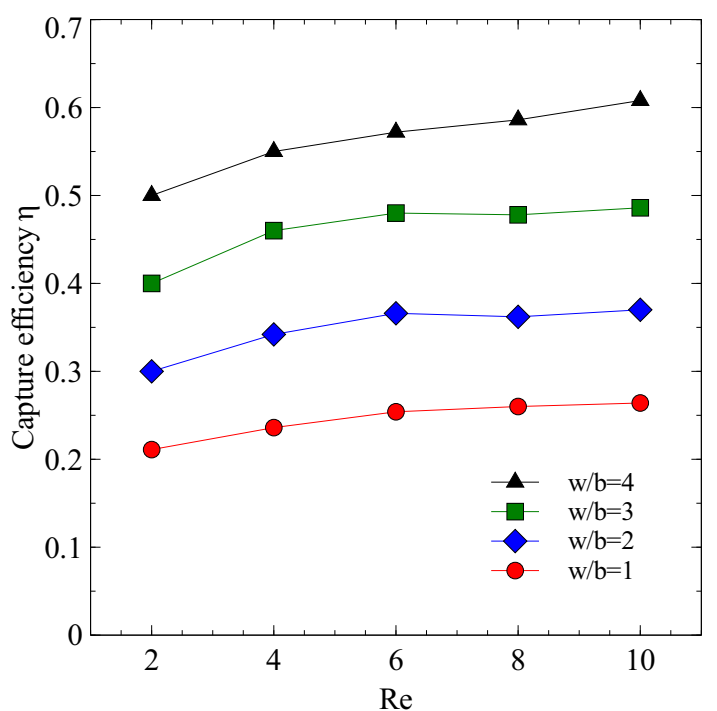

Fig. 10 Capture efficiency as a function of Reynolds number for $\theta=0$.

The effect of orientation angle $\theta$ on the capture efficiency is analyzed. Different orientation angles corresponding to different cross-section area of fiber have significant influence on the capture efficiency. In Fig. 11, capture efficiency as a function of orientation angle is presented for $R e=8$. We can easily recognize that the capture efficiency of square fiber $(b / w=1 / 1)$ at $\theta=\pi / 4$ is higher than for other orientation angles, since $l_{c}$ is maximum for this orientation angle. In addition, the value of capture efficiency of square fiber is symmetrical about $\theta=\pi / 4$. For the aspect ratios of $w / b=2,3,4$, the highest capture efficiency is obtained for $\theta=\arctan b / w$. It is also observed that the capture efficiency for different aspect ratios is very close when the orientation angle is $3 \pi / 8$ due to similar values of $l_{c}$ for this orientation angle, as observed in Fig. 6. More generally, the similarity between Figs. 6 and 11 implies that the capture efficiency is mainly governed by the cross section $l_{c}$.

Figure 12 shows, for $R e=8$, the capture efficiency $\eta$ versus the windward area $l_{c}$ for all aspect ratios $w / b$ investigated in this paper and for different values of orientation angle. Figure 12 illustrates that the larger the cross-section area is, the higher a capture efficiency can be obtained. The capture efficiency increases almost linearly with the cross-section area, which results from the strong inertial force. The particle Stokes number is $S t=\rho_{p} d_{p}^{2} U_{0} /\left(18 \mu D_{h}\right)$, where $\rho_{p}$ is the particle density. With our data, the Stokes number is 
$2.08 \leq S t \leq 8.33$. The injected particles at the upstream of the system exhibit large $S t$ behavior and are essentially uninfluenced by the flow.

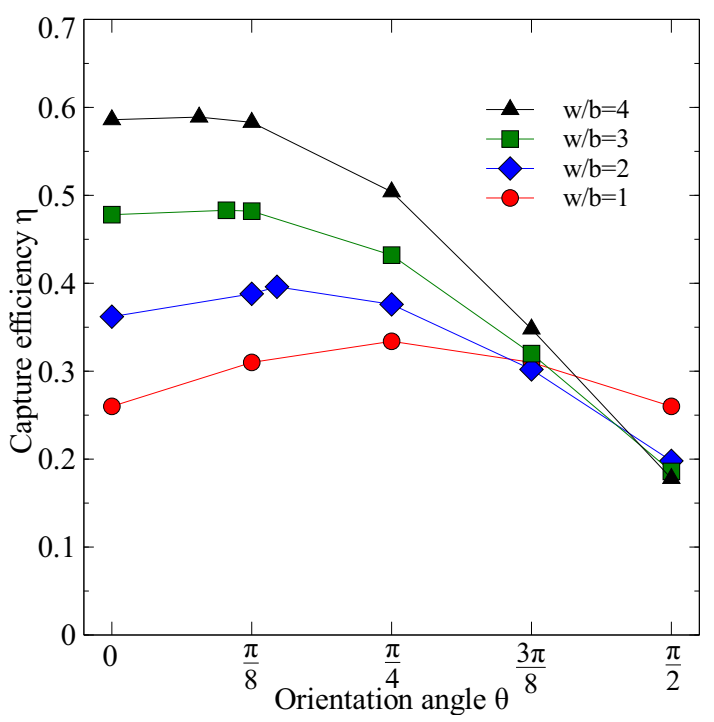

Fig. 11 Capture efficiency as a function of orientation angle for $R e=8$.

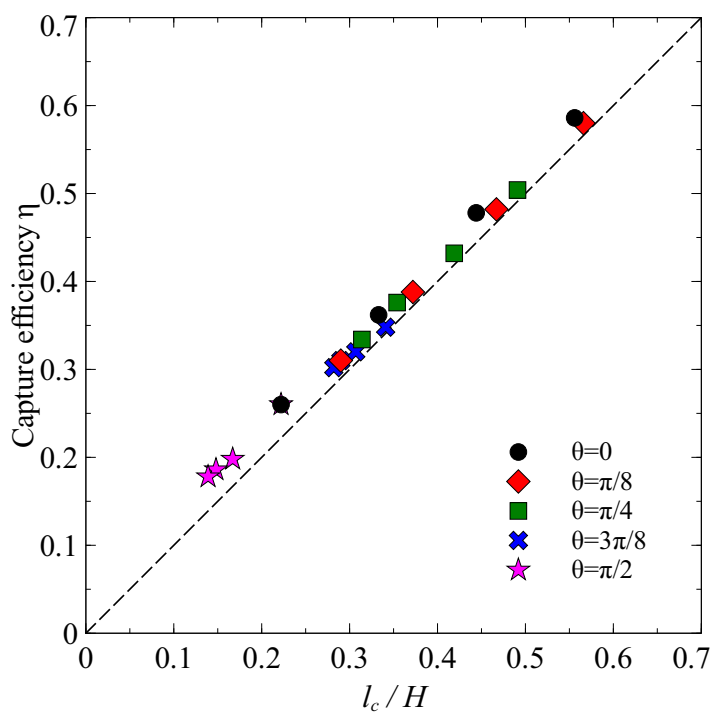

Fig. 12 Capture efficiency as a function of the cross-section area of the rectangular fiber for Re=8 and different orientation angles.

Figure 13 presents the same results for different Reynolds number. The same behavior can be observed, with a small dependency on $R e$, also clearly indicating that the capture efficiency is mainly governed by the windward area $l_{c}$ in this flow regime.

Figure 14 depicts an instantaneous image of the velocity field of the fluid and particle distribution for $S t=8.33$ with different orientation angles $\theta$. It provides an obvious evidence that the particle transport affects the flow field. The link between fluid streamlines presented in Fig. 5 and particle positions presented in Fig. 14 shows that the particles do not follow the streamlines downstream of the fiber. As explained previously, this is due to the inertial regime characterized by large Stokes number as in our simulation. Additionally, since the particle volume is considered in the simulation, a small amount of particles interactions are observed in Fig. 14, though the number of particles within the fluid domain is not huge. 


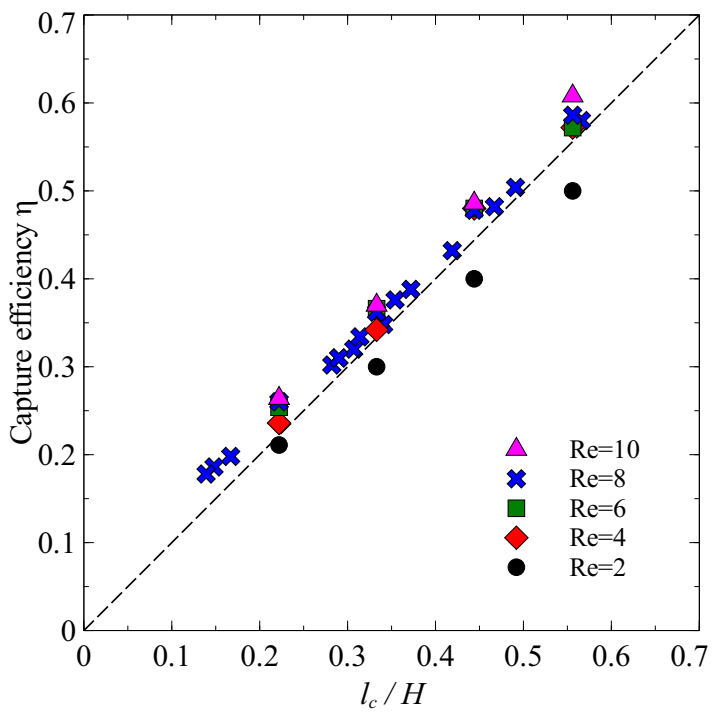

Fig. 13 Capture efficiency as a function of cross-section area of rectangular fiber for different $R e$ and different orientation angles.

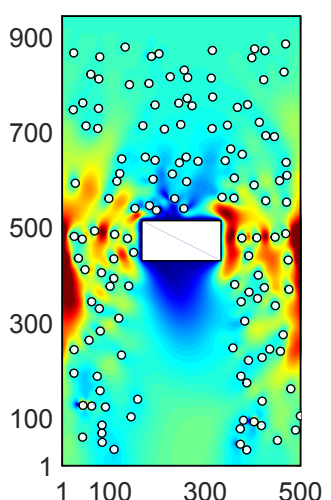

(a)

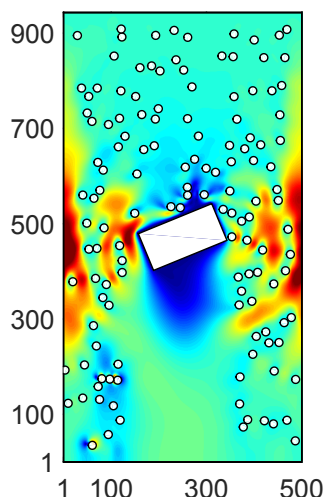

(b)

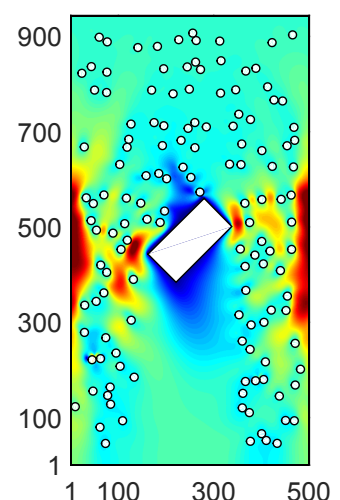

(c)

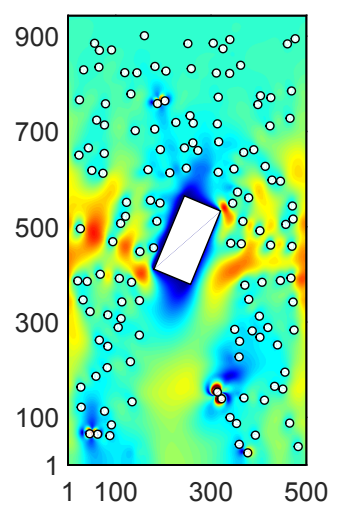

(d)

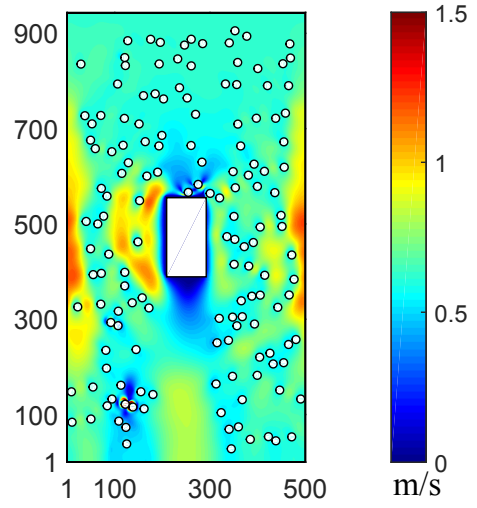

(e)

Fig. 14 Velocity field of fluid and particle distribution for $S t=8.33$ and different value of orientation angle $\theta$.

\subsection{Quality factor}

A best-performing filter first of all not only ensures a high capture efficiency $\eta$ but also maintains a low pressure drop $\Delta p$. In order to judge the performance of fibrous filter, the definition of a quality factor (or the figure of merit) is an indicator of the ratio of penetration rate $P(P=1-\eta)$ to the pressure drop. The quality factor $Q F$ is expressed as $[2,3,50]$ : 


$$
Q F=\frac{-\ln (P)}{\Delta p}
$$

From the filter quality factor standpoint, a larger value of the quality factor $Q F$ indicates a better quality of the fibrous filter.

Figure 15 depicts the relationship between quality factor and Reynolds number with different aspect ratios. It can be observed that the quality factor decreases rapidly when $R e$ varies from 2 to 4 . Then, it gradually changes as $R e$ varies from 4 to 10. Indeed, the pressure drop increases faster than $\eta$, which leads to a decline in the quality factor. As a reference, simulation results for a circular fiber with diameter $D_{f}=D_{h}$ are also presented in Fig. 15. The performance of the filter for $w / b=1$ and $w / b=2$ is approximately equal in the region of $2 \leq R e \leq 10$. They have better quality than the fiber with the aspect ratio of $w / b=3$ and circular fiber. They also show great advantages compared with the case of $w / b=4$.

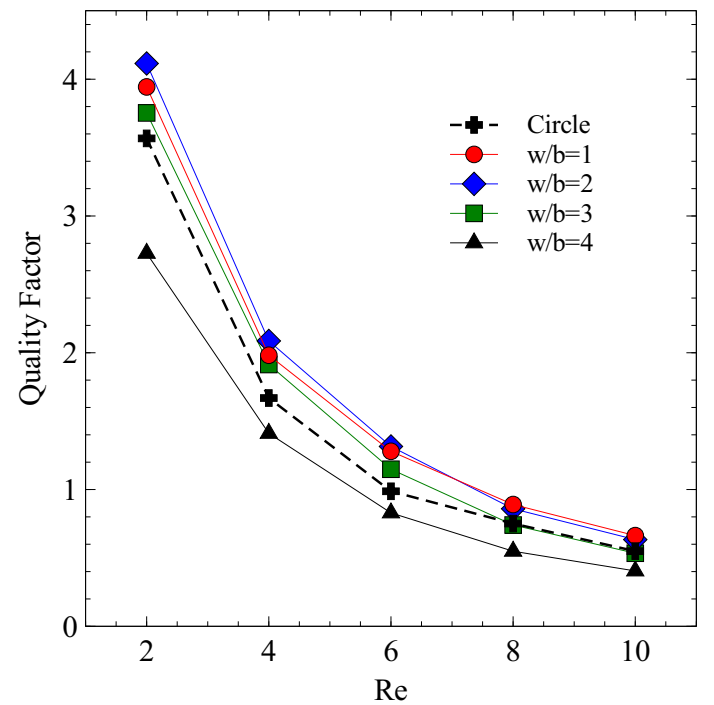

Fig. 15 Quality factor as a function of $R e$ for $\theta=0$.

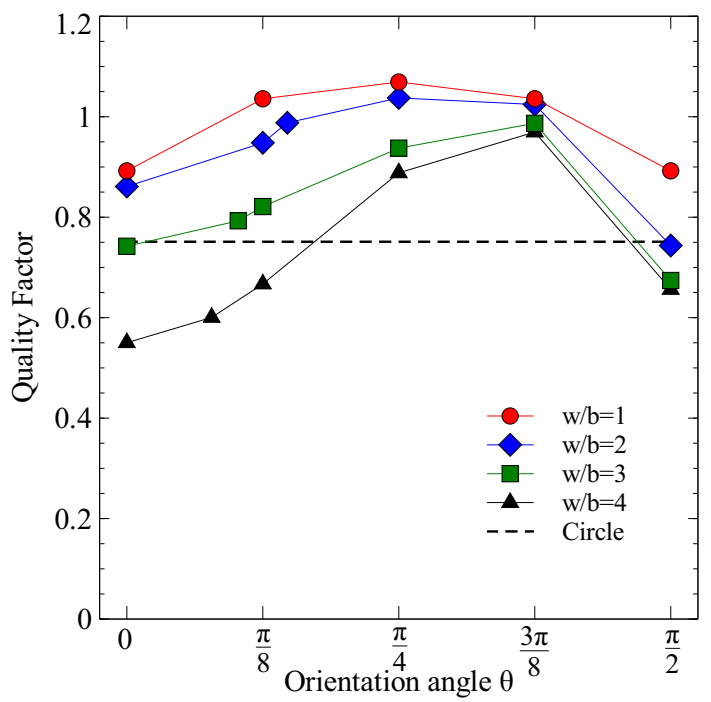

Fig. 16 Quality factor as a function of orientation angle for $R e=8$.

Finally, we explore the effect of orientation angle and aspect ratio for $R e=8$. Figure 16 shows the effect of orientation angle on the quality factor. Figure 16 illustrates that the quality factor for a rectangular fiber increases with the orientation angle at first and then decreases. We find that a higher quality factor can be obtained when $\theta=\pi / 4$ for aspect ratio $w / b=1$. Moreover, for $\theta=3 \pi / 8$, the values of the quality 
factors are quite similar for the different aspect ratio investigated due to the fact that the dimensionless drag force and capture efficiency are not very dependent on $w / b$ for this value of $\theta$. The previous analysis of dependency of drag force of capture efficiency on cross section $l_{c}$ shows clearly that both of them increase with $l_{c}$. In terms of the quality factor, there exists an optimal value of $l_{c} / H$ (around 0.3 ), as illustrated in Fig. 17, that maximizes the quality factor $Q F$. In Fig. 18, we have plotted the $Q F$ as a function of $l_{c} / H$. $Q F$ is strongly influenced by the variation of $l_{c}$. However, it is good for low Reynolds number. Although an increasing Reynolds number induces performance drop, a uniform performance can be obtained.

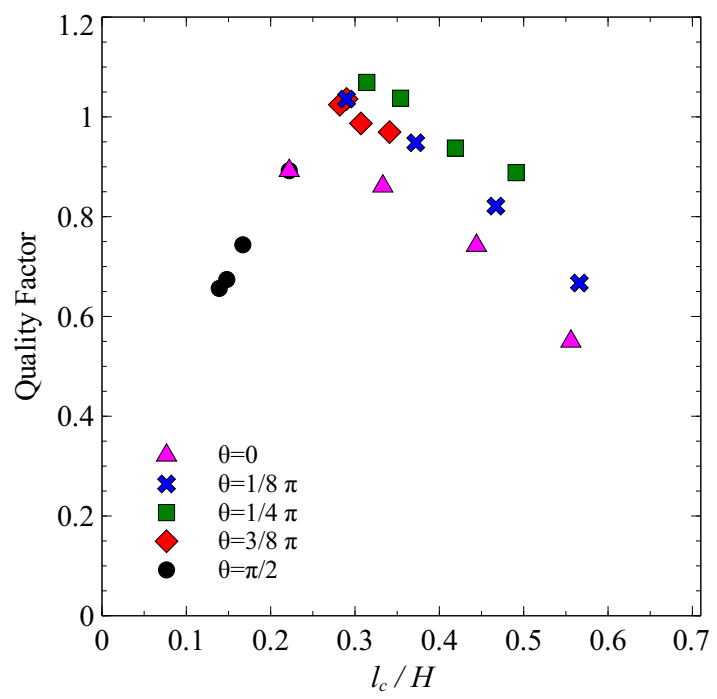

Fig. 17 Quality factor as a function of $l_{c}$ for $R e=8$.

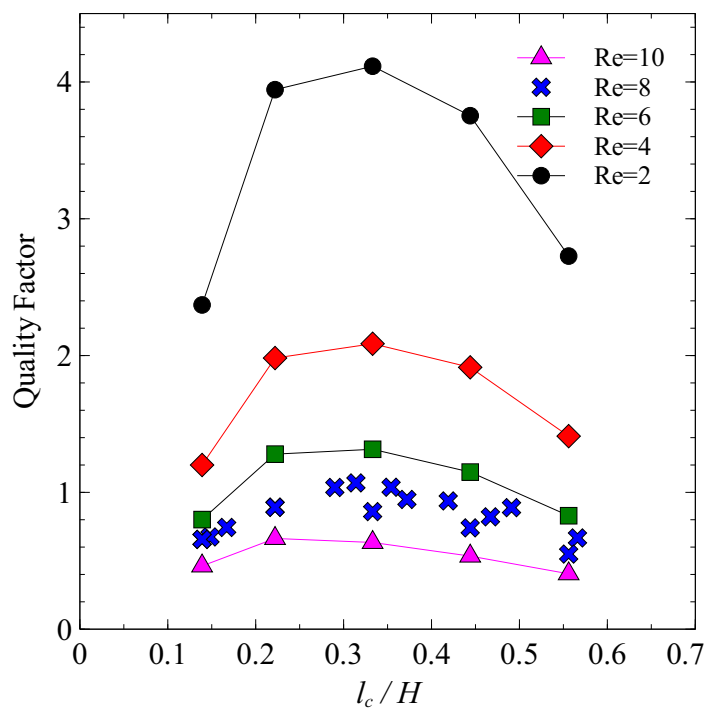

Fig. 18 Quality factor as a function of $l_{c}$ for different $R e$. For $R e=8$, the orientation angles $\theta=0, \pi / 8, \pi / 4,3 \pi / 8, \pi / 2$, for $R e=2,4,6,10, \theta=0$.

\section{Conclusion}

In this study, the filtration processes of fibrous filters with rectangular fibers have been simulated by coupled lattice Boltzmann and discrete element methods. The effects of Reynolds number, aspect ratio and orientation angle on filtration performance parameters, pressure drop and capture efficiency, are investigated for a rectangular fiber during the initial stage of filtration. The quality factor is also used to characterize 
the filtration performance in order to obtain a high capture efficiency and low pressure drop. The following conclusions can be drawn based on the presented simulation results.

First, the dimensionless drag force is dependent not only on the orientation angle but also on the Reynolds number when $2 \leq R e \leq 10$. For large aspect ratio (i.e., $w / b=4$ ), the dimensionless drag force increases rapidly with $R e$. Due to the variation of the windward area $l_{c}$ of the rectangular fiber, the dimensionless drag force decreased greatly with the orientation angle at this aspect ratio. Indeed, it has been illustrated that the drag force increase significantly with $l_{c}$.

Second, the capture efficiency is dependent on both orientation angle and aspect ratio during the filtration process. A larger aspect ratio (i.e., $w / b=2,3,4$ ) at $\theta=0, \pi / 8$ generally leads to a higher capture efficiency. For a square fiber $(w / b=1)$ at $\theta=\pi / 4$, the capture efficiency is higher than for of other orientation angles due to its larger cross-section area. Indeed, we found that for $R e>1$, the capture efficiency increases almost linearly with the windward area. However, it can be noted that the capture efficiency increases slightly with Re. Finally, in terms of quality factor, it is valuable to measure the filtration performance concerning the capture efficiency and pressure drop. For these different aspect ratios, the quality factor increases with orientation angle and reaches its maximum at a certain value and then decreases. The largest quality factor is obtained for square fiber at $\theta=\pi / 4$. Filter performance, through the quality factor, has also been analyzed in terms of windward area. This study shows that there exists an optimal value $l_{c}$ that guarantees the minimization of pressure drop and maximization of capture efficiency.

Generally, through the analysis of pressure drop, capture efficiency and quality factor, the current study provides a contribution for filter design and optimization. Future work will extend the current investigation to more complex studies such as filtration processes with particle loading on fibers implying fiber shape changes. For this, the LB-DE method validated and used in this study will be a valuable tool.

\section{References}

1. Zhu, C., Lin, C.H., Cheung, C.S.: Inertial impaction-dominated fibrous filtration with rectangular or cylindrical fibers. Powder Technology 112, 149-162 (2000)

2. Wang, K., Zhao, H.: The influence of fiber geometry and orientation angle on filtration performance. Aerosol Science and Technology 49, 75-85 (2015)

3. Wang, J., Pui David, Y.H.: Filtration of aerosol particles by elliptical fibers: A numerical study. Journal of Nanoparticle Research 11, 185-196 (2009)

4. Kuwabara, S.: The forces experienced by randomly distributed parallel circular cylinders or spheres in a viscous flow at small reynolds numbers. Journal of the Physical Society of Japan 14, 527-537 (1959)

5. Lee, K.W., Liu, B.Y.H.: Theoretical study of aerosol filtration by fibrous filters. Aerosol Science and Technology 1, 147-161 (1982)

6. Schweers, E., Umhauer, H., Lbffler, F.: Experimental investigation of particle collection on single fibres of different configurations. Particle and Particle Systems Characterization 1, 275-283 (1994)

7. Augusto, L. L. X., Ross-jones, J., Lopes, G. C., Tronville, P., Gonçalves, J. A. S., Krause, M. J.: Microfiber filter performance prediction using a lattice Boltzmann method. Communications in Computational Physics 23(4), 1-22 (2018)

8. Liu, Z.G., Wang, P.K.: Pressure drop and interception efficiency of multifiber filters. Aerosol Science and Technology 26, 313-325 (1997)

9. Stechkina, I.B., Kirsch, A., Fuchs, N.A.: Studies in fibrous aerosol filters iv. calculation of aerosol deposition in model filters in the range of maximum penetration. Annals of Occupational Hygiene $12,1-8(1969)$

10. Fan, J., Lominé, F., Hellou, M.: Numerical study of particle capture efficiency in granular filter. EPJ Web of Conferences 140, 03,003 (2017)

11. Rabiee, B.M., Talebi, S., Abouali, O., Izadpanah, E.: Investigation of the characteristics of particulate flows through fibrous filters using the lattice boltzmann method. Particuology 21, 90-98 (2015)

12. Wang, H., Zhao, H., Guo, Z., Zheng, C.: Numerical simulation of particle capture process of fibrous filters using lattice boltzmann two-phase flow model. Powder Technology 227, 111-122 (2012)

13. Jafari, S., Salmanzadeh, M., Rahnama, M., Ahmadi, G.: Investigation of particle dispersion and deposition in a channel with a square cylinder obstruction using the lattice boltzmann method. Journal of Aerosol Science 41, 198-206 (2010)

14. Hosseini, S.A., Tafreshi, H.V.: On the importance of fibers' cross-sectional shape for air filters operating in the slip flow regime. Powder Technology 212, 425-431 (2011) 
15. Kirsh, V.A.: Stokes flow and deposition of aerosol nanoparticles in model filters composed of elliptic fibers. Colloid Journal 73, 345-351 (2011)

16. Huang, H., Wang, K., Zhao, H.: Numerical study of pressure drop and diffusional collection efficiency of several typical noncircular fibers in filtration. Powder Technology 292, 232-241 (2016)

17. Fardi, B., Liu, B. Y. H.: Flow field and pressure drop of filters with rectangular fibers. Aerosol Science and Technology 17, 36-44 (1992)

18. Fardi, B., Liu, B.Y.H.: Efficiency of fibrous filters with rectangular fibers. Aerosol Science and Technology 17, 45-58 (1992)

19. Wang, C.Y.: Stokes flow through an array of rectangular fibers. International Journal of Multiphase Flow 22, 185-194 (1996)

20. Ouyang, M., Liu, B.Y.H.: Analytical solution of flow field and pressure drop for filters with rectangular fibers. Journal of Aerosol Science 29, 187-196 (1998)

21. Chen, S., Cheung, C.S., Chan, C.K., Zhu, C.: Numerical simulation of aerosol collection in filters with staggered parallel rectangular fibres. Computational Mechanics 28, 152-161 (2002)

22. Filippova, O. and Häanel, D.: Lattice-boltzmann simulation of gas-particle flow in filters. Computers and Fluids 26, 697-712 (1997)

23. Przekop, R., Moskal, A., Gradoń, L.: Lattice-boltzmann approach for description of the structure of deposited particulate matter in fibrous filters. Journal of Aerosol Science 34, 133-147 (2003)

24. Lantermann, Udo and Hänel, Dieter.: Particle monte carlo and latticeboltzmann methods for simulations of gas-particle flows. Computers and Fluids 36, 407-422 (2007)

25. Wang, H., Zhao, H., Wang, K., He, Y., Zheng, C.: Simulation of filtration process for multi-fiber filter using the Lattice-Boltzmann two-phase flow model. Journal of Aerosol Science 66, 164-178 (2013)

26. Lin, K.C., Tao, H., Lee, K.W.: An early stage of aerosol particle transport in flows past periodic arrays of clear staggered obstructions: A computational study. Aerosol Science and Technology 48, 1299-1307 (2014)

27. Ansari, V., Goharrizi, A.S., Jafari, S., Abolpour, B.: Numerical study of solid particles motion and deposition in a filter with regular and irregular arrangement of blocks with using lattice boltzmann method. Computers and Fluids 108, 170-178 (2015)

28. Deng, Y., Liu, Z., Zhang, P., Liu, Y., Wu, Y.: Topology optimization of unsteady incompressible Navier-Stokes flows. Journal of Computational Physics 230(17), 6688-6708 (2011)

29. Deng, Y., Liu, Z.,Wu, J.,Wu, Y.: Topology optimization of steady Navier-Stokes flow with body force. Computer Methods in Applied Mechanics and Engineering 255, 306-321 (2013)

30. De Rosis, A., Falcucci, G., Ubertini, S., Ubertini, F., Succi, S.: Lattice Boltzmann analysis of fluid-structure interaction with moving boundaries. Communications in Computational Physics $13,823-834(2013)$

31. Ernst, M., Dietzel, M., Sommerfeld, M.: A lattice Boltzmann method for simulating transport and agglomeration of resolved particles. Acta Mechanica 224(10), 2425-2449 (2013)

32. Trunk, R., Henn, T., Dörfler, W., Nirschl, H., Krause, M. J.: Inertial dilute particulate fluid flow simulations with an Euler-Euler lattice Boltzmann method. Journal of Computational Science 17, 438-445 (2016)

33. Elghobashi, S.: Particle-laden turbulent flows: direct simulation and closure models. Applied Scientific Research, 48, 301-314 (1991)

34. Elghobashi, S.: On predicting particle-laden turbulent flows. Applied Scientific Research 52(4), 309-329 (1994)

35. Cundall, P.A., Strack, O.: Discrete numerical model for granular assemblies. Geomechanics 29, 47-65 (1979)

36. Lominé, F., Oger, L.: Dispersion of particles by spontaneous interparticle percolation through unconsolidated porous media. Phys. Rev. E 79, 1-12 (2009)

37. Lominé, F., Oger, L.: Transit time during the interparticle percolation process. Physical Review E $82,041,301(2010)$

38. Feng, Y.T., Han, K., Owen, D.R.J.: Coupled lattice Boltzmann method and discrete element modelling of particle transport in turbulent fluid flows: Computational issues. International journal for numberical methods in engineering 72, 1111-1134 (2007)

39. Lominé, F., Scholtès, L., Sibille, L., Poullain, P.: Modeling of fluid-solid interaction in granular media with coupled lattice boltzmann/discrete element methods: application to piping erosion. International Journal for Numerical and Analytical Methods in Geomechanics 37, 577-596 (2013)

40. D'Humières, D., Lallemand, P., Frisch, U.: Lattice gas models for 3d hydrodynamics. Europhysics Letters (EPL) 2, 291-297 (1986)

41. Bhatnagar, P.L., Gross, E.P., Krook, M.: A model for collision processes in gases. I. small amplitude processes in charged and neutral one-component systems. Physical Review 94, 511-525 (1954) 
42. Qian, Y.H., D’Humières, D., Lallemand, P.: Lattice bgk models for navierstokes equation. Europhysics Letters 17, 479-484 (1992)

43. Zou, Q., He, X.: Lattice bgk models for navier-stokes equation. Physics of Fluids 9, 1591-1598 (1997)

44. Ladd, A.J.C.: Numerical simulations of particulate suspensions via a discretized boltzmann equation. part 1. theoretical foundation. Journal of Fluid Mechanics 271, 285-309 (1994)

45. Behrend, O.: Solid-fluid boundaries in particle suspension simulations via the lattice boltzmann method. Phys. Rev. E 52, 1164-1175 (1995)

46. Schwager, T., Poschel, T.: Coefficient of restitution and linear-dashpot model revisited. Granular Matter 9, 465-469 (2007)

47. Verlet, L.: Computer experiments on classical fluids i. thermodynamical properties of lennard-jones molecules. Physical Review 159, 98-103 (1967)

48. Duval, H., Masson, D., Guillot, J.B., Schmitz, P., Humières, D.: Two-dimensional lattice-boltzmann model of hydrosol depth filtration. AIChE Journal 52, 39-48 (2006)

49. Fan, J., Lominé, F., Hellou, M.: Modelling particle capture efficiency with lattice boltzmann method. Communications in Computational Physics 23(4), 1-19 (2017)

50. Kuo, Y.M., Huang, S.H., Lin, W.Y., Hsiao, M.F., Chen, C.C.: Filtration and loading characteristics of granular bed filters. Journal of Aerosol Science 41, 223-229 (2010) 